'School of Dental Sciences, Health Campus, Universiti Sains Malaysia of Science, Malaysia, 16150 Kota Bharu Kelantan.

${ }^{2}$ Forensic Odontology and Oral Blology Unit, Hospital Universiti Sains Malaysia, Health Campus 16150 Kota Bharu Malaysia
Corresponding author: Mohd Fadhli Bin Khamis School of Dental Sciences Health Campus Universiti Sains, Malaysia e-mail: fadhli@usm.my

Editor: Dr Altair A. Del Bel Cury

Received: June 4, 2021

Accepted: October 24, 2021

\section{Validity and reliability of palatal rugae morphometric assessment with 3D laser scanned models}

\author{
Abdullah Abdulkhaleq Alselwi ${ }^{1}$ (D), Mohd Fadhli Bin \\ Khamis $^{1,2}$, Johari Yap Abdullah ${ }^{1}$
}

Aim: To assess the reliability and validity of morphometric features on 3D digital models produced by scanning maxillary dental casts of Malaysian Malay subjects. Methods: Dental casts of 20 subjects were scanned using a 3D laser scanner (Next Engine Inc., Santa Monica, California, USA). The palatal rugae morphometric features were assessed on the resulting 3D models using 3-Matic Research 9.0 software (Materialise NV, Heverlee, Belgium). The assessments were repeated by the first and second authors to assess the intra- and interexaminer reliability, respectively. Rugae morphometric features were also evaluated on the conventional plaster models to assess the validity of the 3D method. Results: Kappa values of the validity ranged from 0.807 to 0.922 for rugae shape, size category and direction. The intraclass correlation coefficient (ICC) for rugae number validity was 0.979 . For intra-examiner reliability, kappa values ranged from $0.716-1.000$ for rugae shape, size category and direction. The ICC for rugae number intra-examiner reliability was 0.949 . Kappa values of interexaminer reliability for rugae shape, size category and direction were $0.723-885$, while the ICC of rugae number was 0.896 . Conclusion: Palatal rugae analyses on 3D digital models scanned by the 3D Next Engine laser scanner using 3-Matic Research 9.0 software are valid and reliable.

Keywords: Forensic dentistry. Forensic anthropology. Palate, Imaging, three-dimensional. 


\section{Introduction}

Palatal rugae are transverse mucosal ridges that are located in the anterior third of the hard palate, behind the incisive papilla, and laterally extended from the median palatine raphe'. The morphometric characteristic of the palatal rugae have been studied by many researchers in many fields of dentistry, especially forensic odontology and orthodontics ${ }^{2-6}$.

Palatal rugae morphometric characteristics might be used for comparison between ante-mortem and post-mortem records for the purpose of human identification in the presence of ante-mortem records such as dental casts, digital images or prosthesis? ${ }^{7}$. They also might be used in conjunction with other methods for sex or ancestry predictions ${ }^{8,9}$.

Plaster dental casts are commonly used by dental clinicians and researchers. However, they are susceptible to damage and occupy physical space for storage $\mathrm{e}^{10,11}$. The digital models offer a solution to the mentioned disadvantages, and they are also ready for superimposition analyses in treatment progress monitoring ${ }^{5,12,13}$ and for sharing with colleagues through the internet ${ }^{11}$.

Realizing the potential roles of digital models, any method to digitize dental casts for digital manipulation must have gone through verification in terms of validity and reliability. Several studies have shown that the difference in linear measurements between the conventional and digital models are within acceptable limits ${ }^{14-16}$.

On the other hand, only limited literatures has reported the analyses on palatal rugae using 3D digital models ${ }^{17-19}$. They used different scanner and software combinations in their studies. The coefficient values were between 0.875 and 1.000 for intraand inter-observer error rates. So far, at least to our best knowledge, there was no quantitative study assessing the validity and reliability of palatal rugae using the $3 \mathrm{D}$ Next Engine laser (Next Engine Inc., Santa Monica, California, USA) scanned models. Thus, we aimed to benchmark the Next Engine laser scanner combined with 3-Matic Research 9.0 (Materialise NV, Heverlee, Belgium), which has been deemed reliable software with other published 3D scanner systems.

\section{Materials and methods}

\section{Sample}

Calculation of the sample size was performed using a web sample size calculator ${ }^{20}$ The inserted values were as follows: minimum acceptable kappa $=0.6$, expected kappa $=0.85$, proportion of outcome for primary rugae $=78.26^{3}$, significance level $=0.05$, power $=80 \%$ and expected dropout rate $=0 \%$. Thus, the estimated sample size was 116 .

This study utilized 20 dental casts that were selected from the School of Dental Sciences archive using a convenience sampling method. The assessments focused on the first three rugae ${ }^{17}$ on each side of the palate. There were 119 palatal rugae observed in the 20 dental casts. Each cast contained at least six rugae in both sides 
of the palate, except for one cast that had five rugae. Dental casts with damaged palatal areas and defects, such as bubbles or porosity, were excluded from the study. Similar to other studies ${ }^{3,9}$, rugae $<2 \mathrm{~mm}$ in length were excluded from this study. Ethical clearance was obtained from the Universiti Sains Malaysia (USM) Human Ethics Committee (USM/JEPeM/19020125).

\section{Data collection}

The dental casts were digitized using the 3D Next Engine laser scanner. The scanning processes were 1) standardized distance between the scanner and the flatter pad on which the cast was stabilized at $14 \mathrm{~cm}$ and 2) a a $360^{\circ}$ scan on the cast from every angle (Figure 1).

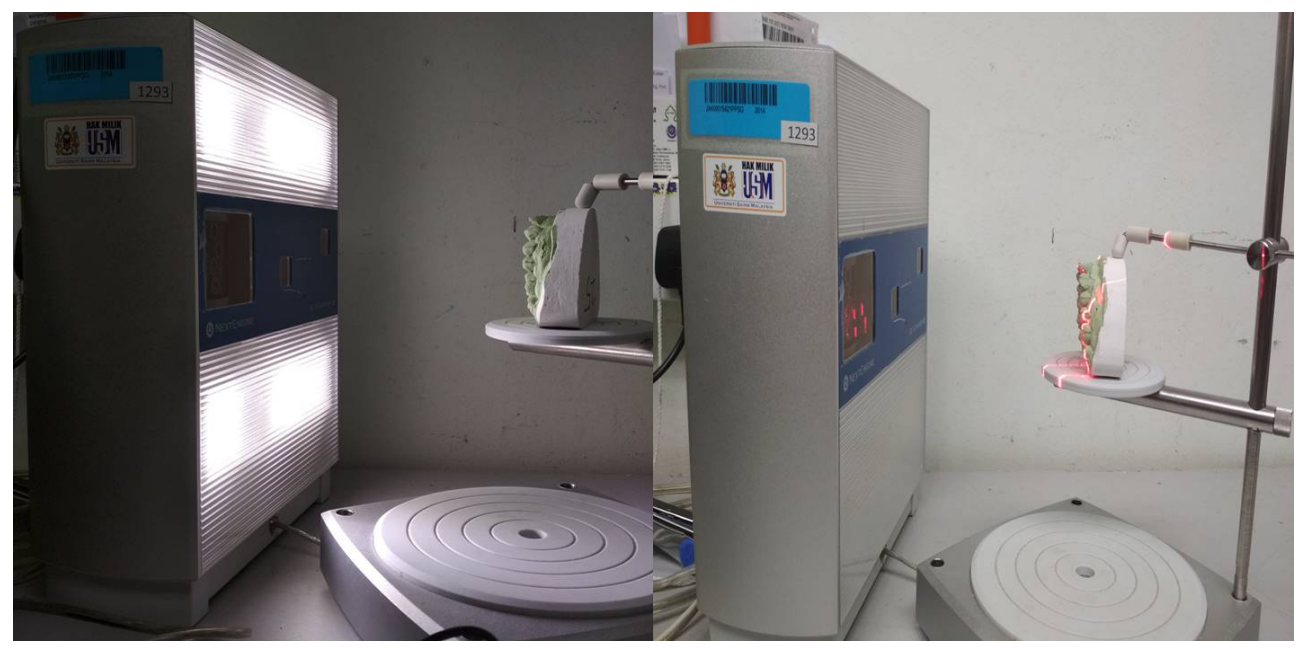

Figure 1. Dental cast scanning using the Next Engine 3D scanner.

The scanning process began with a fluorescent light produced from the scanner capturing a 2D image of the cast, followed by laser beams emitted from the scanner to capture the geometry of the cast (Figure 1). Next Engine scan studio software (Next Engine Inc., Santa Monica, California, USA) stitched single multiple scans together, and the unwanted parts were trimmed out from the field of view to produce the final 3D image. The scanned images were saved as stereolithography (stl) files.

\section{Identification of palatal rugae on the digital models}

Each stl file was opened with Paint 3D version 6.2003.4017.0 (Microsoft, Redmond, WA, USA) software. The palatal rugae were highlighted on 3D digital models ${ }^{17,21}$ using a pixel pen marker tool, which could be changed from 4-6 pixel thickness according to the size and curvature of the rugae to be highlighted (Figure 2). After highlighting the rugae, the rugae shape was determined visually by the researcher. 


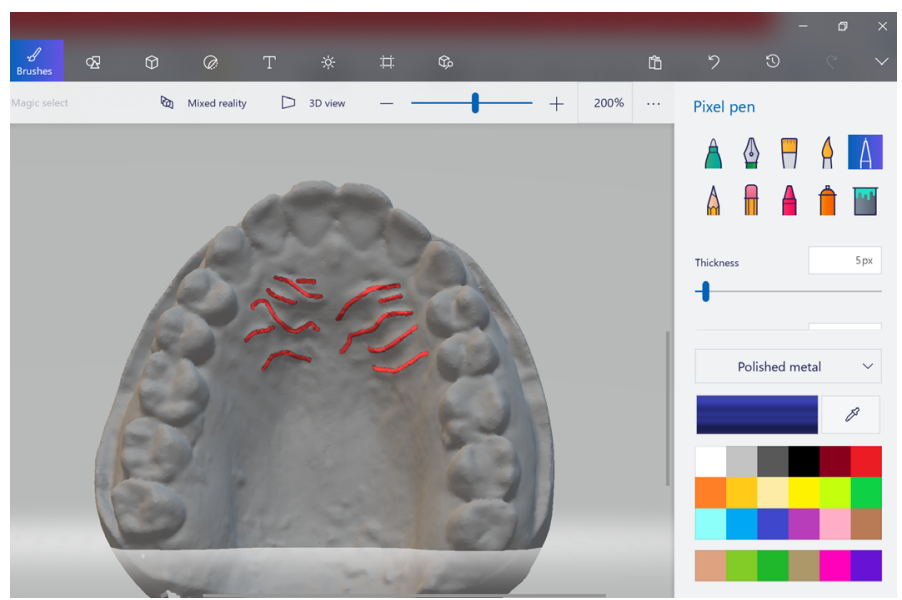

Figure 2. Rugae highlighting on Paint 3D software.

\section{Assessment of palatal rugae on 3D digital models}

The stl file was imported into 3-Matic Research 9.0 software. Each ruga was measured using a length measuring tool in the software accurate to $0.01 \mathrm{~mm}$ which was able to measure the curvatures.

The 3D image was zoomed and angulated as needed using the computer mouse to achieve good visibility of rugae borders during the measuring process.

To assess the rugae size, successive single clicks by the mouse were applied starting at the medial termination point of a ruga following its curvature to the most lateral termination point. A double click was applied at the lateral end point. The measurements of rugae size were in millimetres accurate to $0.01 \mathrm{~mm}$. (Figure 3).

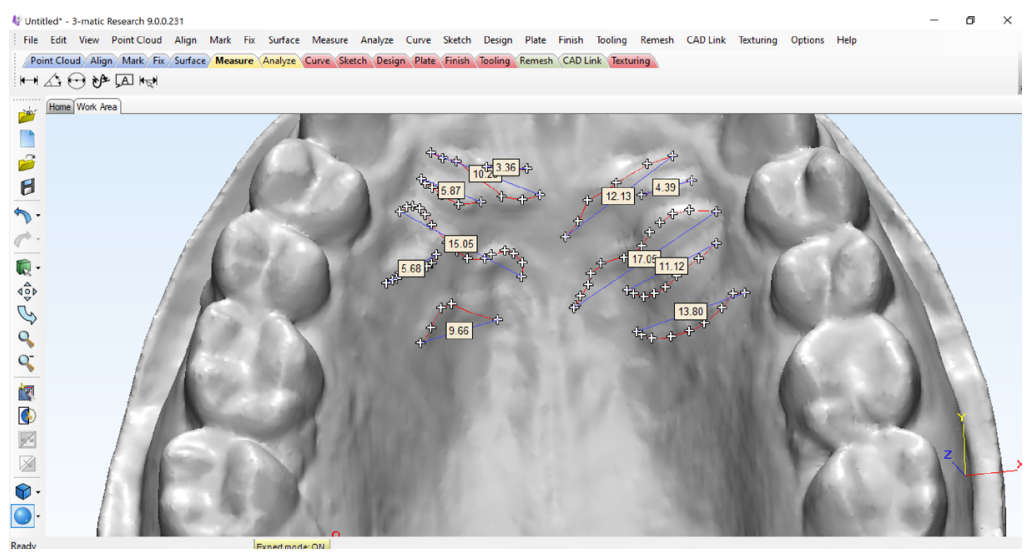

Figure 3. Rugae length measurement on 3-Matic Research software.

The directions of the rugae were also assessed in 3-Matic Research 9.0 software. A straight blue colour line was spontaneously drawn after taking the measurement, 
which connected the medial and lateral point of a measured ruga. The direction of the line from the medial to the lateral end was assessed to determine rugae direction.

The rugae that were $>2 \mathrm{~mm}$ in size were counted on 3-Matic Research 9.0 software to determine the total rugae number of each digital 3D cast.

\section{Assessment of the variables on the plaster models}

The rugae were highlighted directly on the plaster dental casts by the first investigator using a $0.3 \mathrm{~mm}$ lead pencil under an adequate light and glass magnification lens ${ }^{3,9}$. The shape and direction of rugae were evaluated visually by the first investigator (Figure 4).

The rugae size were measured by adapting $0.5 \mathrm{~mm}$ orthodontic wire over each ruga, which was stretched again and measured using a digital calliper accurate to $0.01 \mathrm{~mm}$.

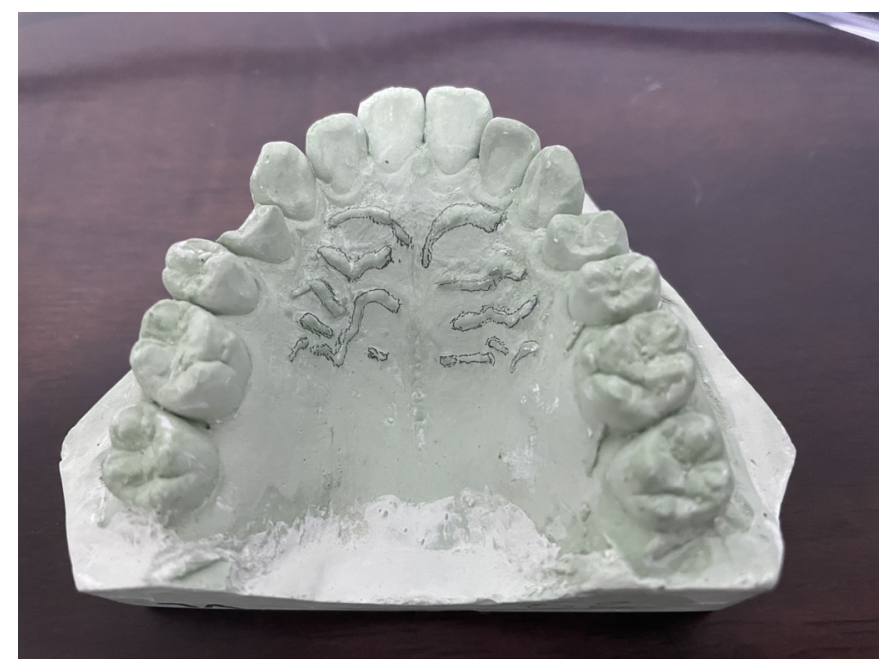

Figure 4. Highlighting the rugae with a pencil on a conventional plaster dental cast

\section{Validity test}

Rugae shape, size, total number and direction on 20 3D digital models were compared statistically with those values derived from the plaster dental casts.

\section{Reliability test}

The first author assessed all variables on the 3D digital models twice within a 1 month interval for intra-examiner reliability. A maximum of four 3D images were evaluated per day. The second author (MFK) assessed the same 3D digital models for inter-examiner reliability (comparison between the first and second authors' measurements).

\section{Statistical analysis}

All the data were imported into SPSS statistical software version 26.0 (IBM, Armonk, NY, USA). Kappa statistics were used to evaluate validity and intra- and inter-exam- 
iner reliability for rugae shape, size category, and direction. The intraclass correlation coefficient (ICC) was used to assess the validity and intra- and interobserver reliability for rugae number. A $P<0.05$ was considered significant.

\section{Results}

Table 1 shows the data for validity evaluation. Kappa values ranged from 0.807 to 0.922 for the shape, from 0.853 to 1.000 for the size category and finally from 0.846 to 1.000 for the direction of rugae.

Table 1. Validity of 3D digital palatal rugae measurements.

\begin{tabular}{lccc}
\hline & Number & Kappa value & P value \\
\hline Rugae 1 shape & 40 & 0.807 & $<0.001$ \\
\hline Rugae 1 Size category & 40 & 1.000 & $<0.001$ \\
\hline Rugae 1 direction & 40 & 0.846 & $<0.001$ \\
\hline Rugae 2 shape & 40 & 0.922 & $<0.001$ \\
\hline Rugae 2 size category & 40 & 0.853 & $<0.001$ \\
\hline Rugae 2 direction & 40 & 0.856 & $<0.001$ \\
\hline Rugae 3 shape & 39 & 0.921 & $<0.001$ \\
\hline Rugae 3 size category & 39 & 0.950 & $<0.001$ \\
\hline Rugae 3 direction & 39 & 1.000 & $<0.001$ \\
\hline
\end{tabular}

Table 2 shows the intra-examiner reliability between the two readings of the first author on the digital 3D models. Kappa values ranged from 0.812 to 1.000 for rugae shape, from 0.889 to 1.000 for rugae size category and from 0.716 to 1.000 for rugae direction.

Table 2. Kappa values for intra-examiner reliability.

\begin{tabular}{lccc}
\hline & Number & Kappa value & P value \\
\hline Rugae 1 shape & 40 & 0.812 & $<0.001$ \\
\hline Rugae 1 Size category & 40 & 0.935 & $<0.001$ \\
\hline Rugae 1 direction & 40 & 0.846 & $<0.001$ \\
\hline Rugae 2 shape & 40 & 0.923 & $<0.001$ \\
\hline Rugae 2 size category & 40 & 0.889 & $<0.001$ \\
\hline Rugae 2 direction & 40 & 0.716 & $<0.001$ \\
\hline Rugae 3 shape & 39 & 1.000 & $<0.001$ \\
\hline Rugae 3 size category & 39 & 1.000 & $<0.001$ \\
\hline Rugae 3 direction & 39 & 0.944 & $<0.001$ \\
\hline
\end{tabular}

Table 3 shows inter-examiner reliability between the two researchers. Kappa values ranged from 0.737 to 0.843 for shape, from 0.785 to 0.857 for size category and from 0.723 to 0.885 for direction. 
Table 3. Kappa values for inter-examiner reliability.

\begin{tabular}{lccc}
\hline & Number & Kappa value & P value \\
\hline Rugae 1 shape & 40 & 0.737 & $<0.001$ \\
\hline Rugae 1 Size category & 40 & 0.857 & $<0.001$ \\
\hline Rugae 1 direction & 40 & 0.846 & $<0.001$ \\
\hline Rugae 2 shape & 40 & 0.843 & $<0.001$ \\
\hline Rugae 2 size category & 40 & 0.785 & $<0.001$ \\
\hline Rugae 2 direction & 40 & 0.723 & $<0.001$ \\
\hline Rugae 3 shape & 39 & 0.841 & $<0.001$ \\
\hline Rugae 3 size category & 39 & 0.843 & $<0.001$ \\
\hline Rugae 3 direction & 39 & 0.885 & $<0.001$ \\
\hline
\end{tabular}

Table 4 shows results for the ICC, which ranged between 0.896 and 0.979 for reliability and validity of number of rugae.

Table 4. Intraclass correlation coefficient of total number counting.

\begin{tabular}{lc}
\hline & Single measure ICC \\
\hline Intra-examiner reliability & 0.949 \\
\hline Inter-examiner reliability & 0.896 \\
\hline Validity & 0.979 \\
\hline
\end{tabular}

The third rugae consistently showed the best kappa values among all the readings with almost perfect reliability and validity.

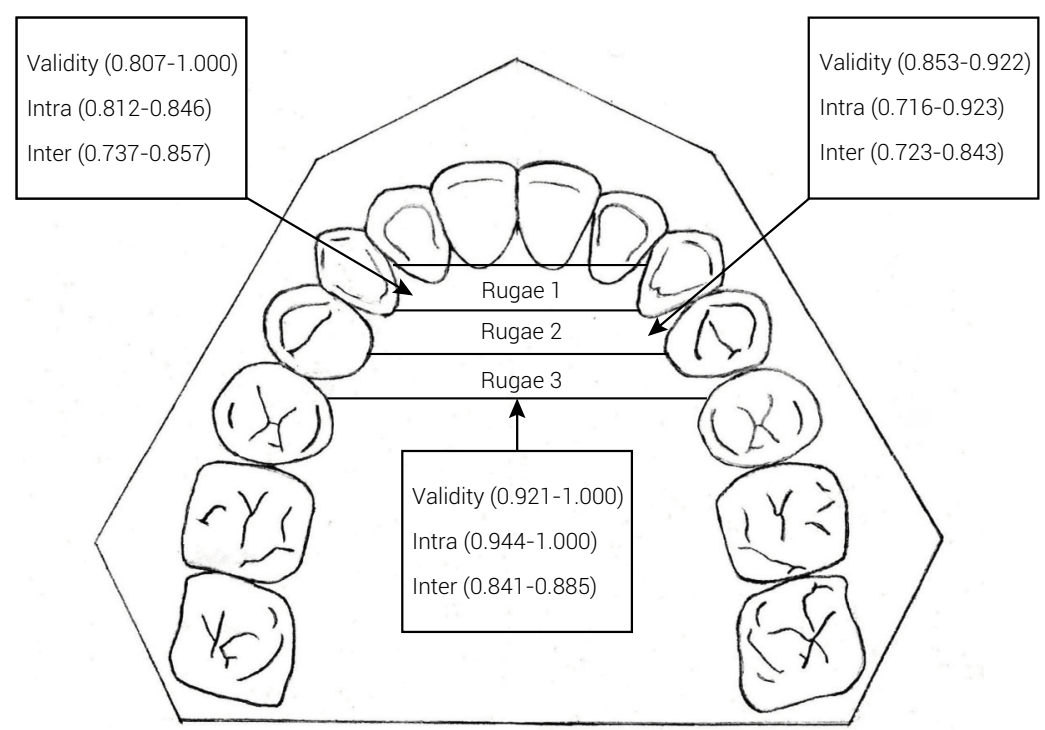

Figure 5. A diagram illustrating kappa values of validity, in addition to intra- and inter-examiner reliability. 


\section{Discussion}

The fundamental of good research is the method should be reliable and valid ${ }^{22}$. The reliability of an instrument is related to its ability to replicate the same measurements under the same conditions (consistency), while the validity of this instrument is related to the reality of the resulting measurements (accuracy) ${ }^{23}$.

A valid tool must be reliable, but a reliable tool may not necessarily be valid. In other words, a wrong measurement may be repeated (highly reliable) but not valid. Thus, we assessed the 3D Next Engine laser scanner and 3-Matic Research 9.0 software from both aspects (i.e., reliability and validity). The focus of reliability assessment was inter- and intra-examiner error study, while validity was assessed by comparing 3D digital model measurements against measurements on plaster dental casts using a pencil, wire, magnifier and digital calliper.

The majority of studies that assessed the palatal rugae have used plaster dental casts. They highlighted the rugae under artificial light and magnification lens using a sharp graphite pencil2,9,24,25 or a pen marker ${ }^{8,26}$. From our point of view, using the sharp pencil allows highlighting a ruga without contacting the adjacent rugae, and the pencil can also be easily erased if there is any mistake. After highlighting the rugae, the shape and direction were visually assessed.

The linear size of the rugae were mostly measured using a digital calliper from the most medial to the most lateral point of each ruga ${ }^{27}$ or using a divider and ruler $^{9,28}$. However, these methods are only able to measure linear distance, excluding the actual curvature distance. Some other studies adapted a flexible metallic wire over the curvature of the rugae to measure the real length of the rugae ${ }^{8,25}$. We were inclined to use the latter method to be more reasonable, as there might be a long ruga with the medial and lateral points close to each other.

The reliability of teeth and dental arch linear measurements on 3D dental casts scanned by the 3D Next Engine laser scanner was assessed by Haque et al. ${ }^{29}$ (2019). They took the linear measurement directly on conventional casts using a digital calliper and also on digital 3D casts using analyses tools in 3-Matic Research 9.0 software. The intra-examiner reliabilities of the two methods were excellent (ICC 0.916-0.995), while the inter-examiner reliability of the digital method was good to excellent (ICC 0.816-0.990). The validity of the digital measurements ranged between 0.913 and $0.996^{29}$.

Few studies have evaluated the morphologic features of palatal rugae on 3D models; however, they only assessed the reliability of rugae size. The reliability of their assessments showed good to excellent ICC values ${ }^{17-19}$.

Choi et al. ${ }^{18}$ (2018) and Barbo et al. ${ }^{17}$ (2018) evaluated morphometric characteristics of palatal rugae on 3D digital casts. In both studies, the reliability of intra-examiner measurements were examined on 30 digital casts. Choi et al. ${ }^{18}$ (2018) found that the ICC was 0.970. While Barbo et al. ${ }^{17}$ (2018) found that, the intra-rater ICC for rugae measurement ranged between 0.999 and 1.00017,18. Another study also reported high coefficient values of intra- and inter-observer measurements using 3D models. The ICC were between 0.875 and 0.957 , respectively ${ }^{19}$. 
Perhaps the reason the previous studies ${ }^{17-19}$ yielded high ICC values is because they assessed the reliability of the length, while in our study, we assessed the reliability and validity of the size category. Moreover, in our study, we assessed the real size of rugae, including their curvatures, not only the linear size from the most medial to the most lateral points of the rugae. The measurement of curvature of each ruga may be more technically sensitive than the measurement of linear ruga length.

The previously mentioned studies ${ }^{17-19}$ did not evaluate the validity of their measurements in comparison to the measurements on conventional plaster models. Saadeh et al. ${ }^{30}$ (2017) reported high correspondence, 0.96 kappa value, of rugae size category on 50 digital 3D models with those on conventional plaster models ${ }^{30}$.

In our study, comparable results were noted. We found that the validity by comparing the rugae morphometric assessment between the digital 3D models and conventional plaster models was almost perfect ${ }^{31}$ and excellent ${ }^{32}$.

As it is clear in the results, the third rugae values were consistently high for validity and reliability. In fact, many studies have used 2D and 3D models to assess the efficacy of palatal rugae as reference points in orthodontics also found that the third rugae were the most stable points, and thus, the most convenient to be used as reference points during superimposition for orthodontic treatment progress evaluation 6,33,34.

The outcome of this study was limited to normal and healthy subjects in which the anatomy of the palate was accessible and had less variation if compared to cleft palate, as an example. Future study should include palatal anomalies where some modification in scanning techniques may need to be explored.

In conclusion, palatal rugae analyses on 3D digital models scanned by the 3D Next Engine laser scanner combined with 3-Matic Research 9.0 software are valid and reliable. The highest values of reliability and validity in both sides of the palate were those of the third palatal rugae.

\section{Acknowledgements}

The authors are grateful to the Ministry of Interior of the Republic of Yemen and Abdullah Bugshan (engineer) and USM Graduate on Time Incentive (1001/PPSG/8123002) for their support and funding for this research. Thanks are due to the Orthodontic Department of Hospital USM for facilitating access to the archives and to Miss Suzana Yahya from the Craniofacial Imaging Laboratory, School of Dental Sciences, USM, for assisting in the scanning process using the 3D Next Engine laser scanner.

\section{Conflict of interest}

The authors have no conflicts of interest to declare.

\section{Data availability}

Datasets related to this article will be available upon request to the corresponding author. 


\section{References}

1. Hauser G, Daponte A, Roberts MJ. Palatal rugae. J Anat. 1989 Aug;165:237-49.

2. Shailaja AM, Romana IRU, Narayanappa G, Smitha T, Gowda NC, Vedavathi HK. Assessment of palatal rugae pattern and its significance in orthodontics and forensic odontology. J Oral Maxillofac Pathol. 2018 Sep-Dec;22(3):430-5. doi: 10.4103/jomfp.JOMFP_190_18.

3. Ahmed AA, Hamid A. Morphological study of palatal rugae in a Sudanese population. Int J Dent. 2015;2015:650648. doi: 10.1155/2015/650648.

4. Gibelli D, De Angelis D, Pucciarelli V, Riboli F, Ferrario VF, Dolci C, et al. Application of 3D models of palatal rugae to personal identification: hints at identification from 3D-3D superimposition techniques. Int J Legal Med. 2018 Jul;132(4):1241-5. doi: 10.1007/s00414-017-1744-X.

5. Dai FF, Liu Y, Xu TM, Chen G. Exploring a new method for superimposition of pre-treatment and post-treatment mandibular digital dental casts in adults. Beijing Da Xue Xue Bao Yi Xue Ban. 2018 Apr;50(2):271-8.

6. Abdel-Aziz HM, Sabet NE. Palatal rugae area: a landmark for analysis of pre- and postorthodontically treated adult Egyptian patients. East Mediterr Health J. 2001 Jan-Mar;7(1-2):60-6.

7. Thomas CJ, van Wyk CW. The palatal rugae in an identification. J Forensic Odontostomatol. 1988 Jun;6(1):21-7.

8. Saraf A, Bedia S, Indurkar A, Degwekar S, Bhowate R. Rugae patterns as an adjunct to sex differentiation in forensic identification. J Forensic Odontostomatol. 2011;29(1):14-9.

9. Pakshir F, Ajami S, Pakshir HR, Malekzadeh AR. Characteristics of palatal rugae patterns as a potential tool for sex discrimination in a sample of Iranian children. J Dent. 2019 Mar;20(1):1-9. doi: 10.30476/DENTJODS.2019.44556.

10. Fleming PS, Marinho V, Johal A. Orthodontic measurements on digital study models compared with plaster models: a systematic review. Orthod Craniofac Res. 2011 2011/02/01;14(1):1-16. doi: 10.1111/j.1601-6343.2010.01503.x.

11. Stevens DR, Flores-Mir C, Nebbe B, Raboud DW, Heo G, Major PW. Validity, reliability, and reproducibility of plaster vs digital study models: comparison of peer assessment rating and Bolton analysis and their constituent measurements. Am J Orthod Dentofacial Orthop. 2006 2006/06/01/;129(6):794-803. doi: 10.1016/j.ajodo.2004.08.023.

12. Anacleto MA, Souki BQ. Superimposition of $3 D$ maxillary digital models using open-source software. Dental Press J Orthod. 2019;24(2):81-91. doi: 10.1590/2177-6709.24.2.081-091.bbo.

13. Lanteri V, Cossellu G, Farronato M, Ugolini A, Leonardi R, Rusconi F, et al. Assessment of the stability of the palatal rugae in a 3D-3D superimposition technique following slow maxillary xpansion (SME). Sci Rep. 2020 Feb;10(1):2676. doi: 10.1038/s41598-020-59637-5.

14. Al-Khatib AR, Rajion ZA, Masudi SaM, Hassan R, Townsend GC. Validity and reliability of tooth size and dental arch measurements: a stereo photogrammetric study. Aust Orthod J. 2012;28(1):22-9.

15. Botticelli S, Pedersen TK, Kuseler A, Norholt SE, Cattaneo PM. Novel 3-D analysis for the assessment of cleft dimensions on digital models of infants with unilateral cleft lip and palate. Cleft Palate Craniofac J. 2019 Jan;56(1):127-33. doi: 10.1177/1055665618770795

16. Nouri M, Asefi S, Baghban AA, Aminian A, Shamsa M, Massudi R. Validity and reliability of a three-dimensional dental cast simulator for arch dimension measurements. Dent Res J. 2014;11(6):656-62

17. Barbo BN, Azeredo F, Menezes LM. Assessment of size, shape, and position of palatal rugae: a preliminary study. Oral Health Dent Stud. 2018;1(1):3. doi: 10.31532/OralHealthDentStud.1.1.003.

18. Choi SH, Koh K, Lee KJ, Hwang CJ, Cha JY. Analysis of the morphological characteristics of the palatal rugae for three-dimensional superimposition of digital models in Korean subjects. BioMed Res Int. 2018 Nov;2018:3936918. doi: 10.1155/2018/3936918. 
19. Saadeh M, Macari A, Haddad R, Ghafari J. Instability of palatal rugae following rapid maxillary expansion. Eur J Orthod. 2017;39(5):474-81. doi: 10.1093/ejo/cjx016.

20. Arifin WN. Sample size calculator (web). 2021 [cited 25 Feb 2021]. Available from: https://wnarifin.github.io/ssc/sskappa.html.

21. De Angelis D, Riboli F, Gibelli D, Cappella A, Cattaneo C. Palatal rugae as an individualising marker: reliability for forensic odontology and personal identification. Sci Justice. 2012 Sep;52(3):181-4. doi: 10.1016/j.scijus.2011.09.002.

22. Kimberlin $\mathrm{CL}$, Winterstein $\mathrm{AG}$. Validity and reliability of measurement instruments used in research. Am J Health Syst Pharm. 2008;65(23):2276-84. doi: 10.2146/ajhp070364.

23. Scribbr. Reliability vs validity: what's the difference? 2019; revised on 2021 Jul 16 [cited 2021 Aug 5]. [Available from: https://www.scribbr.com/methodology/reliability-vs-validity/.

24. Hosmani J, Gadekar NB, Kotrashetti VS, Nayak R, Babji D, Mishra S. Comparison of palatal rugae pattern among Indian and Tibetan population. J Forensic Dent Sci. 2018 Jan-Apr; 10(1):40-4. doi: 10.4103/jfo.jfds_18_16.

25. Jadoon OK, Zaman MU, Zaman FU, Khan D, Farooq U, Seema N, et al. Analysis of palatal rugae pattern in population of Abbottabad: A forensic study. J Ayub Med Coll Abbottabad. 2018 Jul-Sep;30(3):428-37.

26. Dwivedi N, Nagarajappa AK. Morphological analysis of palatal rugae pattern in central Indian population. J Int Soc Prev Community Dent. 2016 Sep-Oct;6(5):417-22. doi: 10.4103/2231-0762.192947.

27. Fatima F, Fida M, Shaikh A. The association between palatal rugae pattern and dental malocclusion. Dental Press J Orthod. 2019;24(1):037e1-e9. doi: 10.1590/2177-6709.24.1.37.e1-9.onl.

28. Rajan VP, John JB, Stalin A, Priya G, Abuthagir AKS. Morphology of palatal rugae patterns among 5-15 years old children. J Pharm Bioallied Sci. 2013;5(Suppl 1):S43-S7. doi: 10.4103/0975-7406.113295.

29. Haque S, Khamis MF, Alam MK, Ahmad WM. The reliability and validity of the measurements in unilateral cleft lip and palate laser scanned 3D dental casts. Pesqui Bras Odontopediat Clín Integr. 2019;19:50-1. doi: 10.4034/pboci.2019.191.137.

30. Saadeh M, Ghafari JG, Haddad RV, Ayoub F. Association among geometric configurations of palatal rugae. J Forensic Odontostomatol. 2017 Jul;1 (35):33-41.

31. Landis JR, Koch GG. The measurement of observer agreement for categorical data. Biometrics. 1977;33(1):159-74. doi: 10.2307/2529310.

32. Koo TK, Li MY. A guideline of selecting and reporting intraclass correlation coefficients for reliability research. J Chiropr Med. 2016 Jun;15(2):155-63. doi: 10.1016/j.jcm.2016.02.012.

33. Shukla D, Chowdhry A, Bablani D, Jain P, Thapar R. Establishing the reliability of palatal rugae pattern in individual identification (following orthodontic treatment). J Forensic Odontostomatol. 2011;29(1):20-9.

34. Hoggan BR, Sadowsky C. The use of palatal rugae for the assessment of anteroposterior tooth movements. Am J Orthod Dentofacial Orthop. 2001 2001/05/01/;119(5):482-8. doi: 10.1067/mod.2001.113001. 